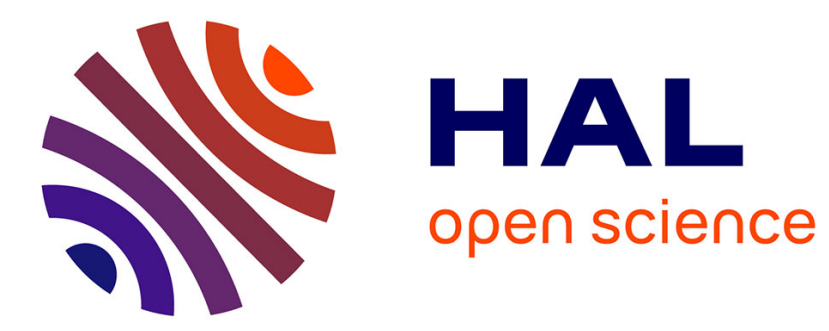

\title{
Cartographie thermique au sein d'écoulements cavitants
} Frédéric Ayela, Damien Colombet, Gilles Ledoux, Olivier Tillement

\section{To cite this version:}

Frédéric Ayela, Damien Colombet, Gilles Ledoux, Olivier Tillement. Cartographie thermique au sein d'écoulements cavitants. La Houille Blanche - Revue internationale de l'eau, 2015, 1, pp.103 - 108. 10.1051/lhb/2015013 . hal-01151735

\section{HAL Id: hal-01151735 \\ https://hal.science/hal-01151735}

Submitted on 13 Jan 2021

HAL is a multi-disciplinary open access archive for the deposit and dissemination of scientific research documents, whether they are published or not. The documents may come from teaching and research institutions in France or abroad, or from public or private research centers.
L'archive ouverte pluridisciplinaire HAL, est destinée au dépôt et à la diffusion de documents scientifiques de niveau recherche, publiés ou non, émanant des établissements d'enseignement et de recherche français ou étrangers, des laboratoires publics ou privés. 


\title{
Cartographie thermique au sein d'écoulements cavitants
}

\author{
Frédéric AYELA ${ }^{1}$, Damien COLOMBET ${ }^{1}$, Gilles LEDOUX², Olivier TILLEMENT²
}

\author{
1. Laboratoire des Ecoulements Géophysiques et Industriels UMR 5519 Université de Grenoble - CNRS, BP 53, 38041 Grenoble Cedex 9, \\ France.e-mail: frederic.ayela@legi.grenoble-inp.fr \\ 2. Institut Lumière Matière UMR 5306 Université Lyon 1-CNRS,69622 Villeurbanne Cedex-e-mail: gilles.ledoux@univ-lyon1.fr
}

\begin{abstract}
RÉSUMÉ. - La maîtrise de la cavitation hydrodynamique au coeur de 'laboratoires sur puces' nous a conduit à développer une métrologie inédite basée sur la sensibilité en température de nanoparticules fluorescentes. Les petites dimensions du système étudié nous ont permis d'effectuer, à l'aide d'un microscope confocal, une cartographie thermique tridimensionnelle de la phase liquide. On a ainsi observé que la transition d'un régime d'écoulement monophasique vers un régime cavitant s'accompagne d'une augmentation de température au cœur de la zone tourbillonnaire. Ce gradient thermique peut s'élever jusqu'à $10^{5} \mathrm{~K} / \mathrm{m}$. Un premier modèle explique cette augmentation par la forte densité de puissance dissipée consécutivement à la transition vers un régime cavitant. Par ailleurs, l'influence d'effets thermosensibles associés à la condensation de la vapeur en aval de l'écoulement doit également être considérée.
\end{abstract}

Mots-clés : cavitation, microfluidique, nanoparticules, fluorescence

\section{Thermal investigation of cavitating flows through microchannels, with the help of fluorescent nanoprobes.}

\begin{abstract}
Thermosensitive fluorescent nanoparticles seeded in de-ionized water have demonstrated the presence of strong thermal gradients in two-phase cavitating microflows. A thermal mapping has been made possible by the use of a confocal microscope, focusing and scanning over the three dimensions of a microchannel interrupted by a microdiaphragm. Working with DI water with flow rates of around 1 liter/ hour, cavitation is here the consequence of high shear rates downstream a diaphragm of hydraulic diameter of $\approx 77 \mu \mathrm{m}$. A thermal gradient $\approx 10^{5} \mathrm{~K} / \mathrm{m}$ has been detected only when the two-phase flow is present. It is located in vortical structures associated with eddies in the shear layers. Thermal profiles have been recorded at different heights of the channel, in the area where the flow exhibits the strongest thermal gradient. The increase of temperature is associated with an increase of the void fraction. That last parameter is reached by considering the spatial variations of the thermally - normalized intensity of the emitting nanoprobes. We believe that overheating is the consequence of the breakdown of large structures into small structures, and of the ultimate conversion of kinetic energy into heat. As the onset of cavitation goes with a drop of the flowrate, a model combining the corresponding hydrodynamic losses to the thermal effects has been established. It demonstrates that the intensity of the thermal gap is enhanced by the microsizes of the device. Meanwhile, the effect of heat transfer due to phase change need to be considered.
\end{abstract}

Key-words: cavitation, microchannel, fluorescence, nanoparticles

\section{INTRODUCTION}

Cet article présente les premiers résultats obtenus grâce à l'utilisation de nanoparticules fluorescentes thermosensibles pour sonder les effets thermiques associés aux écoulements en régime cavitant, que ces derniers soient associés aux dissipations visqueuses, ou aux chaleurs latentes de vaporisation et de condensation des bulles. Connaître les champs de température serait une étape importante pour accéder, à terme, à leur corrélation avec les distributions de vitesse. Cette démarche peut permettre dans un futur proche de clarifier et de sélectionner ensuite les meilleurs modèles en vue d'améliorer la simulation numérique des écoulements cavitants au sein des machines tournantes tels que celles rencontrées dans les industries hydroélectriques ou spatiales. Une approche expérimentale nouvelle sur une géométrie d'écoulement conventionnelle est nécessaire afin de mieux comprendre les mécanismes fondamentaux présents au sein des écoulements cavitants. Une approche basée sur des mesures extérieures, comme par exemple la thermographie infra rouge [Petkovsek et Dular 2013], est limitée aux phénomènes présents seulement sur les bords de l'écoulement. Des mesures non intrusives plus locales, basée sur la technique de la LIF [Rimbert et al., 2013] sont susceptibles de fournir plus d'informations thermiques. Pour progresser dans une orientation de métrologie locale non intrusive, nous avons développé un protocole expérimental inédit associant microscopie confocale, microsystème fluidique et nanoparticules. De par leurs nombreux avantages, les microsystèmes fluidiques constituent donc des systèmes incontournables afin d'accéder à certaines données clés jusqu'à présent difficilement accessibles. La maîtrise de la cavitation hydrodynamique dans des microdiaphragmes dont le diamètre hydraulique égale quelques dizaines de microns nous a permis d'une part de travailler avec de faibles quantités de nanofluide dilué chargé 
en nanoparticules thermofluorescentes, et d'autre part d'inclure ce microsystème fluidique dans un dispositif optique de microscopie confocale qui a permis d'obtenir une cartographie thermique tridimensionnelle au cœur de l'écoulement.

\section{CAVITATION ET MICROSYSTÈMES}

Etudier la cavitation hydrodynamique dans des microcanaux est une démarche extrêmement récente. Avant les travaux de notre équipe publiés en 2011 et 2012 [Medrano et al., 2011, 2012], seuls Mishra et Peles [2005, 2006] avaient, les premiers, atteint un régime cavitant en aval de microdiaphragmes et de microventuris. En effet, la microfluidique est généralement associée à des écoulements laminaires à faible nombre de Reynolds. A travers un diamètre hydraulique inférieur au millimètre, il semble difficile de mettre de tels écoulements dans des conditions favorables à la cavitation. Les injecteurs des moteurs Diesel constituent un cas particulier, étudié depuis plusieurs décennies [Bergwerk, 1959]. Dans un écoulement confiné, on démontre que la chute de pression du liquide consécutive à l'accélération du fluide à travers un étranglement permet d'atteindre d'autant plus facilement la pression saturante que, parmi les deux dimensions caractéristiques d'un microcanal (hauteur $\mathrm{H}$ et largeur w), c'est la plus petite qui varie. Donc, pour des géométries de diaphragmes ou de venturis (rétrécissement puis élargissement à profondeur $\mathrm{H}$ constante), la largeur de l'étranglement $\mathrm{w}$ doit être plus petite que la profondeur du canal. Cela conduit à des gravures résultant d'une attaque profonde réactive anisotrope du silicium, suivant une technologie éprouvée de microfabrication [Medrano et al. 2011]. Le scellement ultérieur d'une plaque de Pyrex préalablement percée permet enfin d'obtenir un microsystème étanche et robuste. Ces microsystèmes fluidiques permettent d'atteindre un régime de cavitation avec un débit maximum de l'ordre du litre par heure.

Par rapport aux installations conventionnelles industrielles, on comprend les potentialités que recèlent ces laboratoires sur puce, que ce soit pour étudier des fluides 'exotiques', rares, toxiques, ou pour développer des métrologies fondées sur les nanotechnologies, technologies puissantes mais inadaptées à l'échelle macroscopique. Cette orientation expérimentale impose d'une part une stricte conservation des lois d'échelle, et d'autre part que la miniaturisation des canaux ne rende pas les effets de surface prépondérants. Nos travaux ont démontré [Medrano et al. 2011, 2012] que tel était le cas dans la quasi totalité des configurations expérimentales étudiées. Les seules observations d'un phénomène spécifiquement attribuable aux petites dimensions du système ont été le changement de la fréquence d'oscillation et de rupture d'une poche de vapeur en aval de microventuris. Par ailleurs, tous les essais ont été effectués avec de l'eau déionisée et filtrée en amont des laboratoires sur puce, dont les surfaces présentent une faible rugosité. Cela a mis en évidence des phénomènes de métastabilité de la phase liquide, phase qui persiste jusqu'à des pressions négatives. Une telle métastabilité s'est avérée être un atout dans les expériences qui seront présentées plus loin, car elle a associé sans équivoque les phénomènes thermiques au régime d'écoulement cavitant. Enfin, il est à noter que le taux de concentration en oxygène dissous, estimé entre $2 \mathrm{ppm}$ et $8 \mathrm{ppm}$, n'a eu aucune influence sur le seuil de déclenchement de la cavitation.

\section{INFLUENCE DES NANOSONDES SUR LE DÉCLENCHEMENT DE LA CAVITATION}

Toute métrologie locale fondée sur l'immersion physique de sondes doit s'affranchir d'effets indirects susceptibles de perturber le phénomène étudié. Dans notre étude, est-ce que la présence de nanoparticules thermosensibles, entités solides dispersées dans l'écoulement, va altérer les phénomènes de cavitation hydrodynamique ? La réponse est a priori négative. Si une particule solide, assimilée à une sphère de rayon $\mathrm{R}$, agit comme un germe de croissance d'une bulle de vapeur, la surpression du gaz par rapport à la pression $P_{L}$ $\mathrm{du}$ liquide environnant sera égale à $\approx 2 \sigma / \mathrm{R}$ où $\sigma=73 \mathrm{mN} / \mathrm{m}$ est la tension de surface de l'eau à température ambiante. Pour des particules millimétriques, cette surpression est négligeable devant la pression du liquide. Pour des nanoparticules, on arrive par contre à la conclusion que la pression dans la poche ne peut tomber sous sa valeur saturante que si la pression du liquide $\mathrm{P}_{\mathrm{L}}$ atteint des valeurs négatives de l'ordre de -80 à -100 bars, valeurs que notre installation expérimentale ne permettait pas d'atteindre. Cette conclusion a été validée expérimentalement pour les écoulements cavitants au travers de microventuris. La présence de nanoparticules de gadolinium fonctionnalisées et rendues hydrophiles par une coque de $\mathrm{SiO}_{2}$, de diamètre nominal égal à $2 \mathrm{~nm}$, et jusqu'à des fractions volumiques solides $\varphi$ (volume de la phase solide / volume total de la suspension) valant $\varphi=10^{-4}$, n'a eu aucun effet sur la métastabilité de la phase liquide. Par contre, au travers de microdiaphragmes, deux régimes ont été distingués en fonction de la fraction volumique $\varphi$ de la phase solide : en dessous de $\varphi=7$. $10^{-5}$ le comportement hydrodynamique du nanofluide est le même que celui du liquide pur, à savoir métastabilité de la phase liquide avant la transition puis une fois le régime diphasique établi, extinction de la cavitation dès lors que la pression en sortie de la zone d'étranglement remonte au dessus de sa valeur saturante. Mais dès lors que $\varphi_{c} \geq 10^{-4}$, le comportement métastable disparaît, la cavitation apparaît dès que la pression du liquide chute sous sa valeur saturante. Tout se passe comme si le liquide était chargé en suspensions de taille submillimétrique. Nous identifions deux causes possibles à ce phénomène : la formation naturelle d'agrégats, mais de par l'estimation de leur taille [Chevalier et al. 2009], cette formation ne saurait expliquer à elle seule la rupture de la métastabilité ; les zones de recirculation en sortie de microdiaphragme, où ces agrégats peuvent s'accumuler et ainsi donner naissance à des structures de taille suffisante pour briser la métastabilité. Les expériences de cartographie thermique avec les nanoparticules fluorescentes ont donc été conduites avec une concentration volumique $\varphi=1,7.10^{-5}$, nettement inférieure à la concentration critique $\varphi_{\mathrm{c}}$ observée.

\section{MONTAGE EXPÉRIMENTAL}

\section{IV.1. Section d'essai hydraulique}

Par rapport aux expériences exclusivement hydrauliques effectuées sur des microsystèmes hybrides, où le canal gravé dans un substrat de silicium était scellé de façon étanche par un capot en pyrex préalablement percé pour l'alimentation fluidique [Medrano et al., 2011], le système testé ici se devait d'être optiquement transparent. On commence ainsi par sceller sur un substrat en Pyrex une feuille de silicium dont l'épaisseur correspondra à la hauteur $\mathrm{H}$ du 
futur microdiaphragme. Par des procédés classiques de lithographie, on duplique le motif final sur le silicium et on le grave sur toute son épaisseur. Le deuxième scellement d'un capot en Pyrex avec ouvertures parachève la réalisation du système Pyrex - silicium - Pyrex optiquement transparent. Il s'agit ici d'un canal horizontal de profondeur $\mathrm{H}=124 \mu \mathrm{m}$, de longueur $\mathrm{L}=3 \mathrm{~mm}$, de largeur $\mathrm{W}=500 \mu \mathrm{m}$, interrompu à mi longueur par un rétrécissement de $\mathrm{w}=56 \mu \mathrm{m}$ sur une longueur $1=100 \mu \mathrm{m}$.

\section{IV.2. Dispositif d'imagerie}

Ce système, une fois connecté, est asservi sur une platine piezo électrique permettant un déplacement dans le plan horizontal XY par pas de $20 \mu \mathrm{m}$ avec une sensibilité de $100 \mathrm{~nm}$, sur des distances de l'ordre de plusieurs $\mathrm{cm}$. La plage de déplacement suivant l'axe vertical $Z$ perpendiculaire au plan est supérieure à $100 \mu \mathrm{m}$, avec une sensibilité nanométrique. L'ensemble est inclus dans l'appareillage d'un microscope confocal modifié pour les besoins de l'expérience. Le schéma expérimental est décrit sur la figure 1 . La source d'excitation est un laser titane - saphir femtoseconde (Tsunami de Spectra Physics), $82 \mathrm{MHz}$, dont la longueur d'onde peut s'accorder entre $400 \mathrm{~nm}$ et $500 \mathrm{~nm}$. Pour les expériences menées ici, sa puissance a été limitée à $30 \mu \mathrm{W}$. Le faisceau est focalisé au cœur de l'écoulement à l'aide d'un objectif à longue distance de travail avec une ouverture numérique de 0,6 et un grossissement de x 20 . On irradie ainsi un volume ellipsoïde (voxel) de diamètres $1 \mu \mathrm{m}$ x $1 \mu \mathrm{m} \times 5 \mu \mathrm{m}$, cette dernière dimension correspondant à la direction verticale perpendiculaire à l'écoulement. Le signal émis par les composés fluorophores traverse l'objectif puis un séparateur de faisceau dichroïque avant d'être focalisé sur un orifice de $20 \mu \mathrm{m}$ de diamètre. La lumière aboutit à l'entrée d'un monochromateur couplé à un dispositif à transfert de charge (EMCCD Newton) qui permet l'analyse spectrale. Pour chaque voxel, le spectre de fluorescence fut intégré pendant un intervalle de mesure de $100 \mathrm{~ms}$. La figure 2 illustre la méthode d'acquisition de chaque voxel.

\section{IV.3. Traceurs et méthode de mesure de la température}

La structure des nanotraceurs employés était la suivante chaque nanoparticule d'or de $5 \mathrm{~nm}$ est enrobée d'une coquille de polysiloxane (épaisseur $25 \mathrm{~nm}$ ) sur laquelle sont greffés environ 400 molécules de fluoresceïne. Les fluoresceines sont incluses dans la couche de polysiloxane comme des dopants dans la couche. C'est pour cela quelles sont

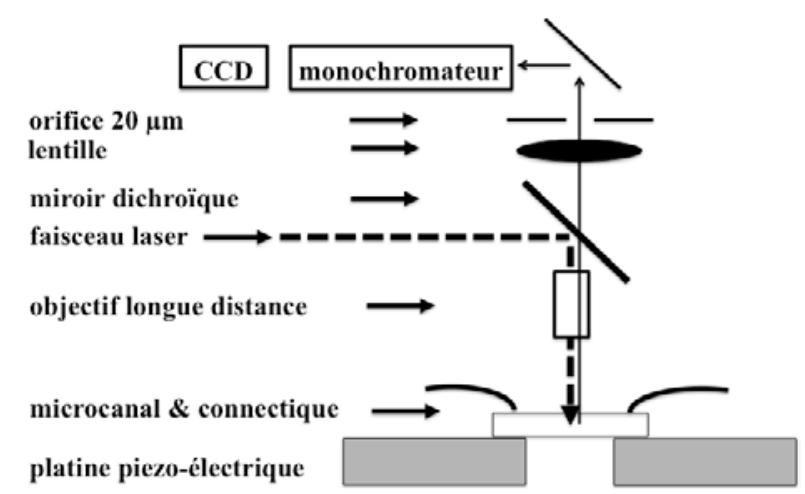

Figure 1 : Schéma expérimental du système de cartographie thermique par microscopie confocale.

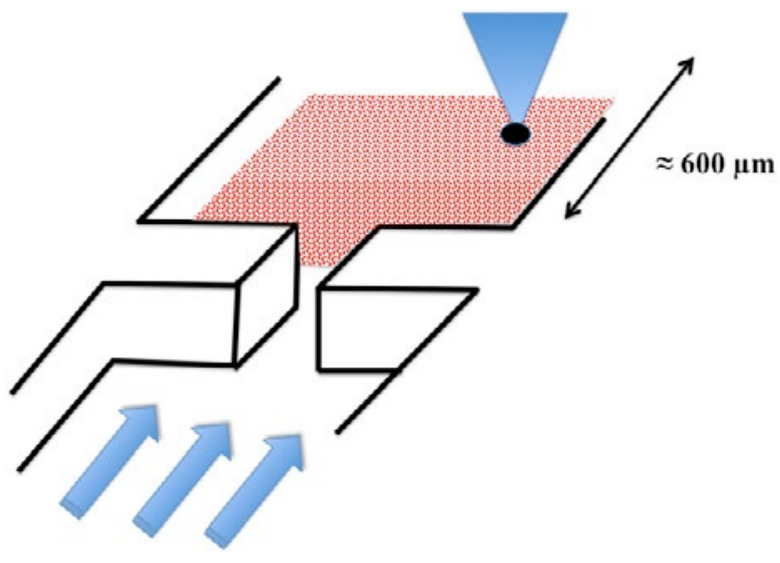

Figure 2 : Vue en perspective de la cartographie d'une strate en sortie de diaphragme, par microscopie confocale.

protégés de l'oxydation et qu'elles sont stables même à relativement haute température $\left(250^{\circ} \mathrm{C}\right)$; les molécules de fluoresceine elles mêmes sont toutes petites $(<<1 \mathrm{~nm})$. Ces nanocomposés hybrides sont ensuite fonctionnalisés et rendus hydrophiles, pour obtenir une bonne stabilité colloïdale. Dans les expériences présentées ici, la fraction volumique de nanoparticules correspond à une occupation moyenne de 5,5 particules par voxel. Ces constituants ont été étalonnés en température, en solution aqueuse de la température ambiante jusqu'à $100^{\circ} \mathrm{C}$, et sous forme de poudre de la température ambiante jusqu'à $600^{\circ} \mathrm{C}$. L'étalonnage consiste à irradier les sondes par un rayonnement de $450 \mathrm{~nm}$, et à observer le spectre émis. Un exemple d'illustration est donné figure 3. On s'intéresse au rapport d'intensité des deux pics principaux. Le principal (rouge) est indépendant de la température, ce qui permet d'affirmer que le rapport calculé s'affranchit des fluctuations d'intensité de la source d'excitation. L'intensité du second pic (bleu) diminue lorsque la température augmente, et le rapport 'bleu' sur 'rouge' permet de remonter à la valeur de la température. La sensibilité relative de ce paramètre est de $6.10^{-3}{ }^{\circ} \mathrm{C}^{-1}$, entre $10^{\circ} \mathrm{C}$ et $60^{\circ} \mathrm{C}$.

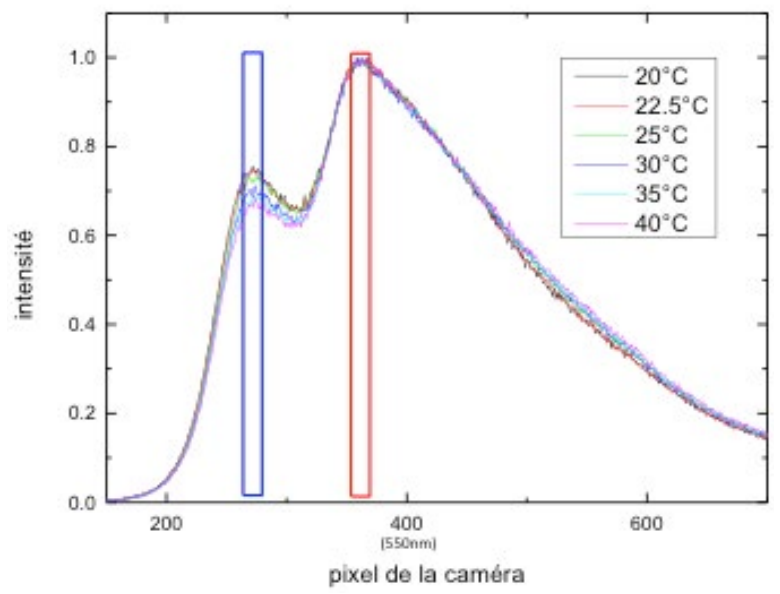

Figure 3 : Spectre d'émission des fluorophores. Le rapport d'intensité entre la bande (rouge) indépendante de la température et celle (bleue) thermosensible permet de determiner la temperature, indépendament des fluctuations d'intensité de la source d'excitation. 


\section{RÉSULTATS}

\section{V.1. Comportement hydrodynamique}

La cartographie thermique de l'écoulement s'analyse conjointement à ses caractéristiques hydrauliques. En régime d'écoulement liquide, il existe une relation quadratique simple entre la différence de pression $\Delta \mathrm{P}$ aux bornes d'un microsystème et le débit volumique $\mathrm{Q}_{1} . \Delta \mathrm{P}=\rho \mathrm{Q}_{1}{ }^{2} /$ $\left(2 \mathrm{C}_{\mathrm{A}}^{2} \mathrm{~W}^{2} \mathrm{H}^{2}\right)$ où le coefficient de décharge $\mathrm{C}_{\mathrm{A}}$ vaut ici $\mathrm{C}_{\mathrm{A}}=$ 0,93 et $\rho=1000 \mathrm{~kg} / \mathrm{m}^{3}$, masse volumique de l'eau considérée comme constante sur la plage de températures considérée. Le coefficient de décharge (sans unité) est l'inverse d'un coefficient de pertes de charge.

Dans notre configuration expérimentale, nous imposons la valeur de $\Delta \mathrm{P}$ et nous mesurons le débit. Ici, l'écoulement débouche à $\mathrm{P}_{\text {atm }}$; la mise en pression en amont se fait en appliquant la pression, via un gaz neutre, sur la surface extérieure d'une poche souple renfermant une grande quantité de liquide. Cette membrane étanche prévient de toute dissolution de gaz dans le liquide et le grand volume permet de conserver à une valeur constante la valeur de $\Delta \mathrm{P}$ pendant l'expérience. Lorsque le débit excède une valeur critique $\mathrm{Q}_{\text {cav }}$ qui dépend de la géométrie de l'écoulement (ici $\mathrm{Q}_{\text {cav }}=200 \mu \mathrm{l} / \mathrm{s}$ ), la pression en sortie de diaphragme tombe sous la valeur $\mathrm{P}_{\text {sat }}=2200 \mathrm{~Pa}$ attendue à température ambiante, et la transition vers un régime liquide - vapeur est susceptible de se produire. Comme indiqué précédemment, l'absence de germes solides et l'absence de rugosité des parois du microsystème permettent de continuer d'augmenter le débit tout en conservant une phase liquide métastable. La transition vers un régime cavitant est alors susceptible d'intervenir inopinément (ici à $\mathrm{Q}_{1}=227 \mu \mathrm{l} / \mathrm{s}$ ) ; elle se traduit par une diminution du débit de $\mathrm{Q}_{1}$ à $\mathrm{Q}_{1-\mathrm{v}}$ et donc à une diminution du coefficient de décharge de $C_{A}$ vers $C_{B}=0,89$. Une fois le régime diphasique établi, l'écoulement obéit à la relation $\Delta \mathrm{P}=\rho \mathrm{Q}_{1-\mathrm{v}}{ }^{2} /\left(2 \mathrm{C}_{\mathrm{B}}{ }^{2} \mathrm{~W}^{2} \mathrm{H}^{2}\right)$.

La figure 4 illustre, dans un diagramme $\Delta \mathrm{P}(\mathrm{Q})$, ces deux régimes. Des observations optiques [Medrano et al. 2012] ont démontré qu'alors le jet liquide émergent du diaphragme déviait arbitrairement sur une paroi latérale, créant ainsi une zone de recirculation tourbillonnaire, avec des taux de cisaillement très élevés créant des zones de dépression locales à l'origine de la cavitation. Ces zones, où la pression reste inférieure à celle du jet émergent, expliquent que la cavitation perdure un peu quand $\mathrm{Q}_{1-\mathrm{v}}$ diminue sous la valeur critique $\mathrm{Q}_{\text {cav }}$ s'atténuant avant de disparaître ici lorsque $\mathrm{Q}<174 \mu \mathrm{l} / \mathrm{s}$ à $\Delta \mathrm{P}=3,8$ bars.

\section{V.2. Cartographie thermique}

Le volume dont nous présentons la cartographie thermique a été scruté en aval du diaphragme. Six plans ont été balayés aux cotes $\mathrm{z}=10 \mu \mathrm{m}, \mathrm{z}=36 \mu \mathrm{m}, \mathrm{z}=50 \mu \mathrm{m}, \mathrm{z}=74 \mu \mathrm{m}$, $\mathrm{z}=98 \mu \mathrm{m}, \mathrm{z}=114 \mu \mathrm{m}(0<\mathrm{z}<\mathrm{H}=124 \mu \mathrm{m})$. On rappelle que chaque voxel est enregistré pendant $100 \mathrm{~ms}$, qu'il y a environ 750 voxels par plan et que la durée d'enregistrement d'une image est de quelques minutes (acquisition et déplacement de la platine). Cette mesure permet donc d'accéder au champ température moyenné en temps. Les images de la figure 5 ont été enregistrées avec $\mathrm{Q}_{1-\mathrm{v}}=216 \mu \mathrm{l} / \mathrm{s}$ à $\Delta \mathrm{P}=6,2$ bars, consécutivement à la transition vers un régime cavitant. La variation locale de température va de $14{ }^{\circ} \mathrm{C}$ à $27{ }^{\circ} \mathrm{C}$.

Une photographie optique de la zone cartographiée est également présentée, où les zones sombres correspondent

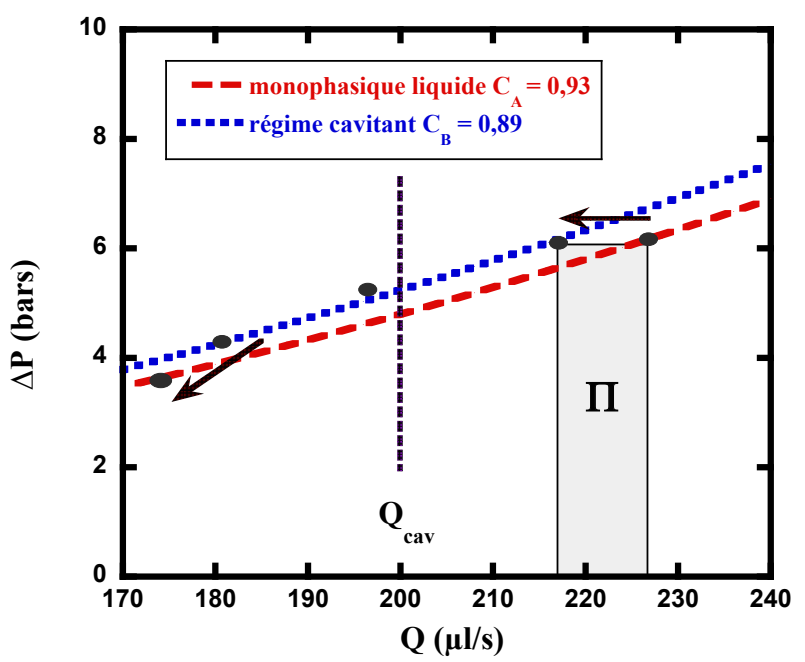

Figure 4 : Relation expérimentale $\Delta P(Q)$. Au dessus d'une valeur critique $Q_{\text {cav }}$, le regime d'écoulement monophasique liquide devient métastable et la transition vers un régime cavitant est alors susceptible de se produire. Cette transition se traduit par une diminution du débit à pression constante. La surface balayée est homogène à une puissance, notée П.

à la phase liquide et les zones claires à la phase vapeur. L'analyse de ces cartographies nous fournit les enseignements suivants.

La strate la plus basse $(\mathrm{z}=10 \mu \mathrm{m})$ présente une zone de température à peu près uniforme, où on distingue néanmoins un infime refroidissement à l'emplacement suivi par le jet. Le deuxième plan $(\mathrm{z}=36 \mu \mathrm{m})$ laisse entrevoir l'amorce d'une zone de gradient thermique (isothermes $15{ }^{\circ} \mathrm{C}$ et $16{ }^{\circ} \mathrm{C}$ dans la zone de recirculation à gauche du jet, isotherme $14^{\circ} \mathrm{C}$ au niveau du jet émergeant). Ce contraste thermique se confirme et s'étend, dans le plan $\mathrm{z}=50 \mu \mathrm{m}$ ainsi que dans le plan $z=74 \mu \mathrm{m}$. Un léger sous refroidissement $\left(13^{\circ} \mathrm{C}\right)$ se généralise dans les zones de recirculation mais est plus marqué à la sortie du diaphragme à $\mathrm{z}=98 \mu \mathrm{m}$, tandis que la zone d'échauffement gagne en intensité. L'élévation de température est maximale à $\mathrm{z}=114 \mu \mathrm{m}$ avec un point chaud mesuré à $26{ }^{\circ} \mathrm{C}$ alors que la température dans le jet liquide évolue, dans ce plan, entre $16^{\circ} \mathrm{C}$ et $17{ }^{\circ} \mathrm{C}$. Dans ce dernier plan, situé à proximité de la limite supérieure du canal, les zones de refroidissement ont disparu.

Ce gradient thermique est associé à la cavitation. L'évolution thermique de l'écoulement est ensuite présentée sur la figure 6 dans le plan fixe $\mathrm{z}=114 \mu \mathrm{m}$, pour des différences de pression de plus en plus faibles. A la diminution du débit correspond une nette atténuation du contraste thermique et la zone 'chaude' piégée dans la recirculation gauche disparaît pour $\Delta \mathrm{P}=3,8$ bars avec l'extinction de la cavitation. Une autre démonstration expérimentale de la corrélation entre gradient thermique et cavitation a été la suivante. Le microscope a été focalisé au point où les mesures précédentes avaient présenté la plus grande élévation de température, et une mesure en temps réel a été lancée, sous une différence de pression de $\Delta \mathrm{P}=6,2$ bars. Pendant environ 3 minutes, l'écoulement reste dans une phase liquide métastable à une température moyenne de $20^{\circ} \mathrm{C}$. Cette valeur peut s'expliquer par les frottements visqueux. Lorsque la cavitation survient, la température augmente instantanément à $26^{\circ} \mathrm{C}$ ainsi que l'amplitude des fluctuations. Ainsi, l'origine des gradients 


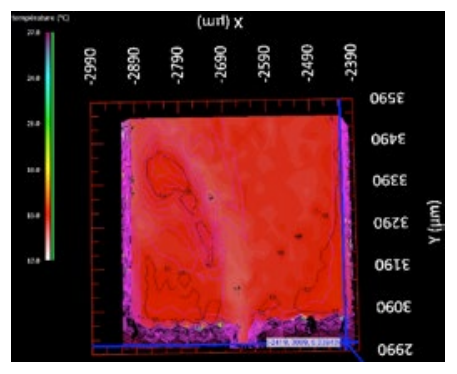

$\mathrm{Z}=10 \mu \mathrm{m}$

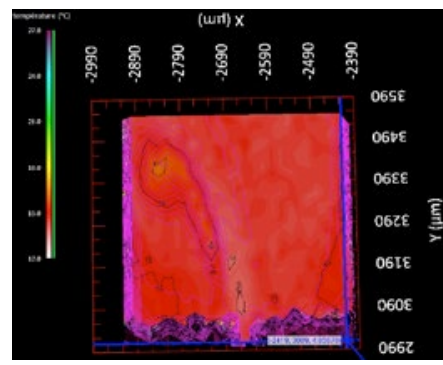

$\mathrm{z}=36 \mu \mathrm{m}$

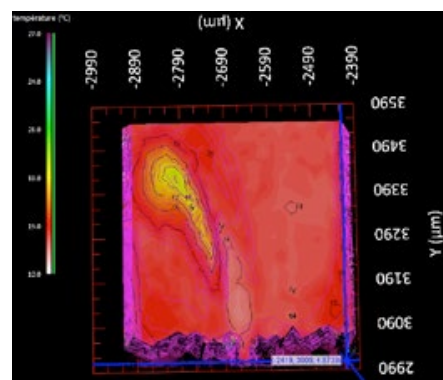

$\mathbf{z}=\mathbf{5 0} \boldsymbol{\mu m}$

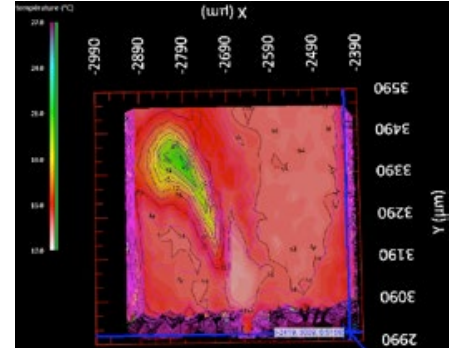

$\mathrm{z}=74 \mu \mathrm{m}$

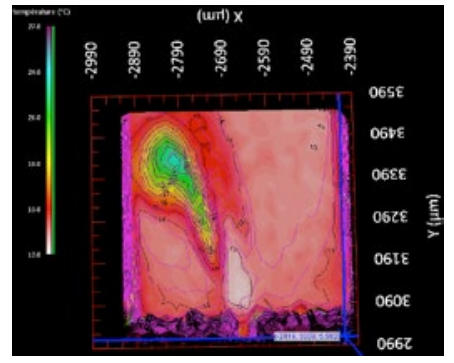

$\mathrm{Z}=98 \mu \mathrm{m}$

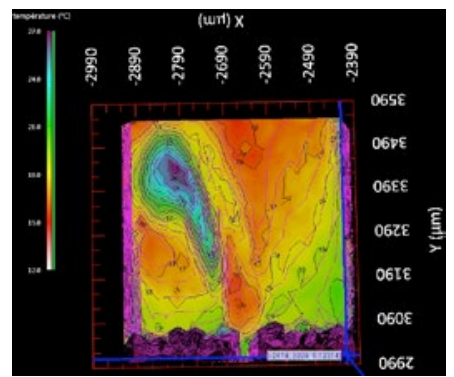

$\mathrm{Z}=114 \mu \mathrm{m}$

Figure 5 : Cartographies thermiques en aval d'un microdiaphragme de hauteur $H=124 \mu \mathrm{m}$. Ces images ont été enregistrées en différents plans de l'écoulement avec un débit $Q_{l-v}=216 \mu \mathrm{l} / \mathrm{s}$ et avec $\Delta P=6,2$ bars, consécutivement à la transition vers un régime cavitant.

thermiques est bien à rechercher dans la physique du régime cavitant et non pas uniquement dans la simple dissipation visqueuse d'un écoulement monophasique liquide.

\section{V.3. Taux de vide}

Une autre information accessible par ces enregistrements est relative au taux de vide. L'intensité de chacun des deux pics luminescents réémis dépend du nombre de nanoparticules fluorescentes. Puisque les particules sont hydrophiles, une diminution globale d'intensité représente donc une diminution de la phase liquide. Une analyse de cette information, pour $\mathrm{Q}=227 \mu \mathrm{l} / \mathrm{s}$ à $\Delta \mathrm{P}=6,2$ bars, a démontré que l'élévation de température maximale du liquide s'accompagnait d'une augmentation du taux de vide. Enfin, elles ont également permis de savoir que, contrairement à ce qu'une seule observation optique pouvait laisser croire, la zone de plus fort taux de vide était confinée d'une part latéralement et d'autre part sur la demi hauteur supérieure du canal.

Ainsi, nous avons identifié un gradient volumique de température de l'ordre de $10^{5} \mathrm{~K} / \mathrm{m}$ qui est la signature du régime cavitant. Le paragraphe suivant développe deux modèles susceptibles d'expliquer ce gradient ; un modèle dit 'de perte de charge' et un modèle dit 'effets thermosensibles'.

\section{ANALYSE}

\section{VI.1. Echauffement dû aux effets visqueux.}

Le modèle dit de pertes de charge s'appuie sur l'analyse graphique, dans un diagramme $\Delta \mathrm{P}(\mathrm{Q})$, de la transition vers le régime de cavitation (figure 4). De même que sur un diagramme de Clapeyron $\mathrm{P}(\mathrm{V})$ la surface balayée lors d'une transformation représente de l'énergie mécanique, la surface balayée par la représentation de la diminution du débit de $\mathrm{Q}_{1}$ à $\mathrm{Q}_{1-\mathrm{v}}$ à $\Delta \mathrm{P}$ constant dans un diagramme $\Delta \mathrm{P}(\mathrm{Q})$, est homogène à une puissance $\Pi=\Delta \mathrm{P} .\left(\mathrm{Q}_{1}-\mathrm{Q}_{1-\mathrm{v}}\right)$. Cette diminution de puissance hydraulique exprime que le fluide gagne à être dans une phase liquide métastable, et que l'écoulement en régime diphasique coûte un prix qui se traduit par une diminution du débit. Notre modèle stipule que la puissance $\prod$ est absorbée sous forme calorifique par le liquide et que cette absorption est à l'origine de l'augmentation de température. Cette absorption est localisée là où les zones tourbillonnaires les plus larges induites par la phase vapeur se fractionnent en structures de plus en plus petites jusqu'à ce que leur énergie cinétique soit dissipée par la viscosité.

Nous définissons la densité volumique de puissance $\mathrm{p}=\prod /(\mathrm{SH})$ où $\mathrm{S}$ est la surface horizontale délimitant la 


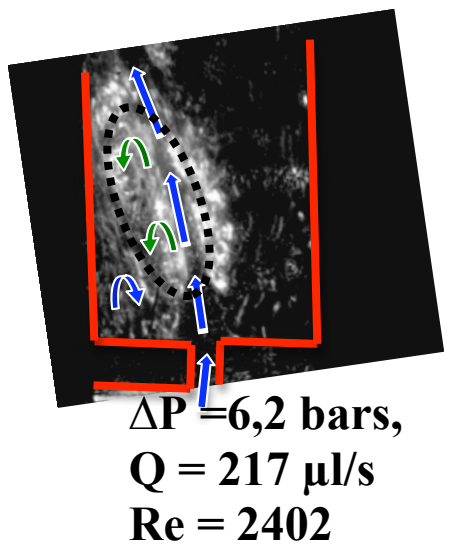

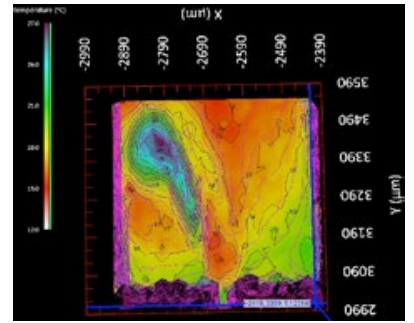

$\Delta \mathbf{P}=\mathbf{6 , 2}$ bars,

$\mathrm{Q}=217 \mu \mathrm{l} / \mathrm{s}$

$\operatorname{Re}=\mathbf{2 4 0 2}$

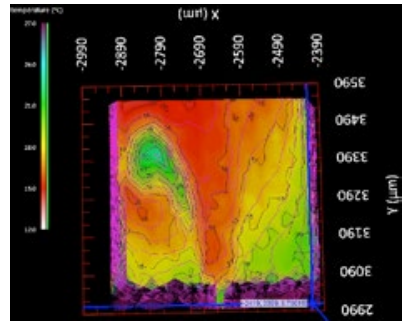

$\Delta P=5,4$ bars,

$\mathrm{Q}=196 \mu \mathrm{l} / \mathrm{s}$

$\operatorname{Re}=\mathbf{2 1 7 1}$

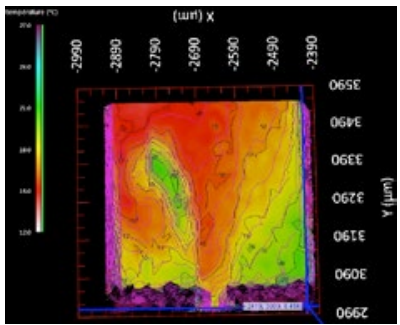

$$
\begin{aligned}
& \Delta P=4,2 \text { bars, } \\
& Q=180 \mu \mathrm{l} / \mathrm{s} \\
& \operatorname{Re}=1994
\end{aligned}
$$

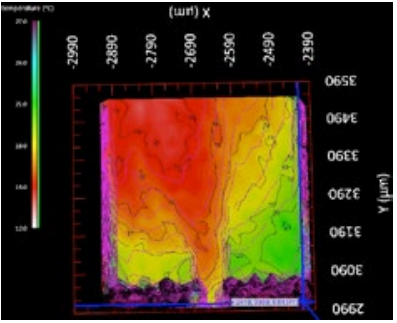

$$
\begin{aligned}
& \Delta P=3,8 \text { bars, } \\
& Q=174 \mu \mathrm{l} / \mathrm{s} \\
& \operatorname{Re}=1925
\end{aligned}
$$

Figure 6 : Cartographies enregistrées à $z=114 \mu \mathrm{m}$, pour des valeurs décroissantes de $\Delta P$. La photographie à gauche est un enregistrement optique. La cavitation disparait à $\Delta P=3,8$ bars.

zone d'absorption. D'après l'illustration de la figure 5 , nous pouvons dans un premier temps estimer que $\mathrm{S} \approx(\mathrm{W} / \mathrm{n})^{2}$ où $n$ est compris entre 3 et 5 . En faisant l'hypothèse que nous n'avons qu'un phénomène de transfert par conduction (c'est à dire que l'on néglige l'évacuation de la chaleur par convection du jet liquide) et que les faces supérieure et inférieure du canal sont à la même température constante $\theta_{0}$ (évacuation de la chaleur suivant la direction verticale $\mathrm{z}$ ), l'équation de la chaleur $\mathrm{d}^{2} \theta / \mathrm{d}^{2} \mathrm{z}=-\mathrm{p} / \mathrm{k}$ et les conditions aux limites fournissent :

$$
\theta-\theta_{0}=\Delta \theta=(p / 2 k) \cdot z(H-z)
$$

où $\mathrm{k}$ est la conductivité thermique de l'eau en phase liquide.

L'équation (1) indique que l'augmentation maximale de température est atteinte à $\mathrm{z}=\mathrm{H} / 2$ et en développant l'expression de $\mathrm{p}$ on arrive à :

$$
\Delta \theta_{\max }=\frac{1}{128} \frac{C_{A}-C_{B}}{C_{B}^{2}} \frac{\mu^{3} \operatorname{Re}^{3}}{k \rho^{2}} \frac{(w+H)^{3}}{w^{2} W^{2} H} n^{2}
$$

où le nombre de Reynolds est ici calculé dans le diaphragme.

L'équation (2) souligne l'importance des effets de taille associés aux dimensions du microcanal. En effet, pour une valeur donnée du nombre de Reynolds l'écart de température augmente quand $\mathrm{w} / \mathrm{H}->0$. Ce modèle explique l'augmentation notable de la température par la forte concentration volumique de puissance dans un microvolume. Pour que l'équation (2) fournisse $\Delta \theta=0$ quand la cavitation disparaît pour $\mathrm{Q}<\mathrm{Q}_{\mathrm{cav}}$, il faut compléter le modèle en exprimant qu'alors $C_{B} \rightarrow C_{A}$. Ce développement, valable sur une plage de débit très étroite, ne sera pas fait ici.

La figure 7 compare l'élévation de température mesurée à celle calculée à partir de différentes valeurs de $\mathrm{n}$ dans

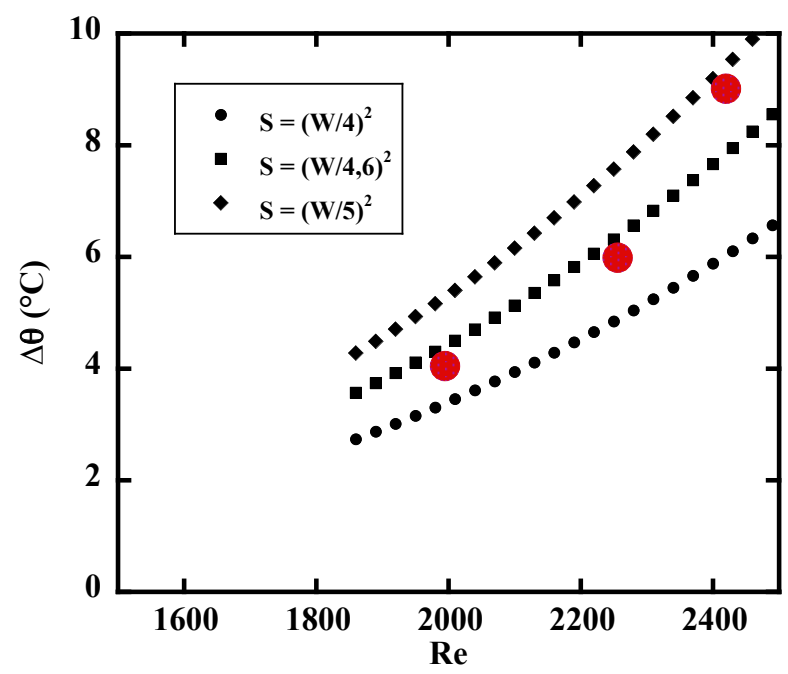

Figure 7 : Comparaison des valeurs expérimentales d'échauffement maximum avec celles prédites par l'équation (2), pour différentes valeurs de surface de localisation. 
l'équation (2). L'intervalle $4,5<\mathrm{n}<5$ correspond à une situation cohérente au vu de l'écoulement.

\section{VI.2. Echauffement dû au changement de phase}

Une seconde explication à l'élévation de la température de la phase liquide réside dans les processus de changement de phase. En effet, la température de la phase liquide peut être augmentée localement sous l'effet des effets visqueux mais également sous l'effet de la condensation de la phase gaz. Il est possible qu'une partie des bulles de vapeur soit piégée par la recirculation en aval du diaphragme, y séjournant un temps suffisant pour finalement se condenser complètement, dégageant alors une certaine quantité de chaleur dans la phase liquide.

L'équation de conservation de l'enthalpie totale de la phase liquide pour un volume de contrôle donné nous montre qu'il existe principalement deux termes sources. Le premier est généré par dissipation due aux effets visqueux au sein de l'écoulement. Alors que le second correspond au transfert interfacial d'enthalpie causé par le changement de phase et peut s'exprimer simplement comme le produit entre $\Gamma_{L}$ flux de masse de vapeur transféré et $\left(h_{L}+U_{L}{ }^{2} / 2\right)$ l'enthalpie totale du liquide. $\Gamma_{\mathrm{L}}$ dépend directement du taux de vaporisation du liquide ou de condensation de la vapeur.

En considérant que la cavitation a lieu en régime thermique et en négligeant les transferts de chaleur en phase gaz, le terme de transfert de masse peut s'écrire en fonction d'un coefficient de transfert de chaleur $H_{L}$, de l'aire interfaciale d'échange par unité de volume $\mathrm{a}_{\mathrm{i}}$, des enthalpies de chacune des phases et de la différence de température entre la phase gaz et la phase liquide $\left(\theta_{\mathrm{G}}-\theta\right)$ comme suit [Colombet et al. 2010]

$$
\Gamma_{L}=\frac{a_{i} H_{L}\left(\theta_{G}-\theta\right)}{h_{G}-h_{L}}
$$

où la température de la phase gaz est égale à la température de saturation basée sur la pression locale $\theta_{G}=\theta_{\text {sat }}(P)$ qui pour l'eau peut être directement donnée par les tables de propriétés thermodynamiques.

Par conséquent, pour un écoulement stationnaire, dans le centre de la recirculation où la vitesse du liquide s'annule et où le temps de séjour des bulles est le maximum, si nous négligeons les effets visqueux ainsi que la diffusion laminaire et turbulente de la chaleur, le bilan d'enthalpie nous montre que le terme de transfert de masse doit quasiment s'annuler soit $\Gamma_{\mathrm{L}}=0$. Ainsi, selon l'expression de $\Gamma_{\mathrm{L}}$ (Eq. 3), au centre de la zone tourbillonnaire, la température de la phase liquide doit se rapprocher de celle de la phase gaz tel que $\theta=\theta_{\mathrm{G}}=\theta_{\text {sat }}\left(\mathrm{P}_{\mathrm{L}}\right)$. Comme le montre la figure 5, localement au centre de la recirculation, la température maximale de la phase liquide est comprise entre $\theta=\theta_{\text {sat }}=21^{\circ} \mathrm{C}$ et $27^{\circ} \mathrm{C}$. Ce qui correspond à une pression statique locale de $\mathrm{P}_{\mathrm{L}}=2400-3500 \mathrm{~Pa}$.

Sachant que la pression en sortie a été fixée à $\mathrm{P}_{\text {out }}=1,013$ bar, cette faible valeur de pression correspond à des coefficients de pression $\mathrm{Cp}=\left(\mathrm{P}_{\mathrm{L}}-\mathrm{P}_{\text {out }}\right) / \mathrm{DP}$ négatifs compris entre $\mathrm{Cp}=-0.15$ et -0.23 . Cet ordre de grandeur apparait comme en accord avec les simulations numériques réalisées précédemment par notre groupe pour un écoulement d'eau à travers un diaphragme d'une géométrie très similaire [Pellone et al. 2010] $(1=100 \mu \mathrm{m}, \mathrm{w}=60 \mu \mathrm{m}$, Fig. 8-c2, $-0.35<\mathrm{Cp}<-0.10)$. Afin d'étudier plus en détail l'évolution spatiale des taux des transferts de masse et de chaleur induit par le changement de phase, de plus amples investigations sont nécessaires. Il serait notamment intéressant de chercher à accéder simultanément aux champs de température, de vitesse et de fraction volumique de gaz dans le but d'obtenir une cartographie du terme de transfert masse au sein d'un écoulement cavitant $\Gamma_{\mathrm{L}}$.

\section{CONCLUSION}

Dans ce travail, une technique de mesure de température par fluorescence induite a été développée afin de mesurer la température de la phase liquide au sein d'un écoulement cavitant. Ces premières mesures, réalisées pour un écoulement d'eau déionisée à travers un micro-diaphragme, démontrent l'existence de fort gradient de température $\left(10^{5} \mathrm{~K} / \mathrm{m}\right)$ dans certaines zones de l'écoulement. Un échauffement est notamment observé dans la recirculation formée en aval du diaphragme. La cause de cette élévation de température semble s'expliquer soit par l'augmentation des effets visqueux amplifiée par les petites dimensions du système fluidique, soit par la présence d'une forte condensation de la phase gaz (vapeur). Des expériences complémentaires en cours devraient permettre de clarifier la contribution de ces deux apports possibles de chaleur ( $\varphi$ et $\Gamma_{\mathrm{L}}$ ). Il ne fait aucun doute que l'étude des écoulements cavitants à micro-échelle couplée à des techniques avancées de mesure s'avère très prometteur pour mieux comprendre les mécanismes présents au coeur des écoulements cavitants.

\section{REFERENCES}

BERGWERK W. (1959) - Flow pattern in diesel nozzle spray holes. Proceedings of the Institute on Mechanical Engineers. 173 (25) : 655-660

Chevalier J., Tillement O., Ayela F. (2009) - Structure and rheology of $\mathrm{SiO} 2$ nanoparticle suspensions under very high shear rates. Phys. Rev. E 80 : 051403

Colombet D., Goncalves E., Fortes-Patella R. (2010) Simulation de la cavitation non isotherme avec un modèle de transport de taux de vide. 2léme Congrès Français de Mécanique, Bordeaux, 26 au 30 août 2010

Medrano M., Zermatten P.J., Pellone C., Franc J.P., Ayela F. (2011) - Hydrodynamic cavitation in microsystems. I. Experiments with deionized water and nanofuids. Phys. Fluids. $23: 127103$

Medrano M., Pellone C., Zermatten P.J., Ayela F. (2012) Hydrodynamic cavitation in microsystems. II. Simulation and optical observations. Phys. Fluids. 24 : 047101

Mishra C., Peles Y. (2005) - Cavitation in flow through a micro-orifice inside a silicon microchannel. Phys. Fluids. 17 : 013601

Mishra C., Peles Y. (2006) - An experimental investigation of hydrodynamic cavitation in micro-venturis. Phys. Fluids. 18 : 103603

Pellone C., Franc J.P., Ayela F., Medrano M. (2010) - Ecoulement non cavitant et cavitant dans un micro-diaphragme. 12ème Journée de l'Hydrodynamique, Nantes, 17-19 Novembre 2010

Petkovsek M., Dular M. (2013) - IR measurements of the thermodynamic effect in cavitating flow. Int. J. Heat Fluid Flow. $44: 756-763$

Rimbert N., Castanet G., Funfaschilling D. (2013) Measurement of thermal effects in a cavitating channel flow by 2cLIF. 8th International Conference on Multiphase Flow ICMF 2013, Jeju, Korea, May 26-31 2013 